\title{
Polyhydroxyalkanoate granules quantification in mixed microbial cultures using image analysis: Sudan Black B versus Nile Blue A staining
}

\author{
Daniela P. Mesquita ${ }^{a}$, A. Luís Amaral ${ }^{\mathrm{a}, \mathrm{b}}$, Cristiano Leal $^{\mathrm{b}}$, Adrian Oehmen ${ }^{\mathrm{c}}$, \\ Maria A.M. Reis ${ }^{c}$, Eugénio C. Ferreira ${ }^{a, *}$ \\ ${ }^{a}$ CEB - Centre of Biological Engineering, Universidade do Minho, Campus de Gualtar, 4710-057 Braga, Portugal \\ ${ }^{b}$ Instituto Politécnico de Coimbra, ISEC, DEQB, Rua Pedro Nunes, Quinta da Nora, 3030-199 Coimbra, Portugal \\ ' CQFB/REQUIMTE, Departamento de Química, Faculdade de Ciências e Tecnologia, Universidade Nova de Lisboa, 2829-516 Caparica, Portugal
}

\section{A R T I C L E I N F O}

\section{Article history:}

Available online 14 January 2015

\section{Keywords:}

Image analysis

Enhanced biological phosphorus removal

(EBPR) systems

Mixed microbial cultures (MMC)

Polyhydroxyalkanoates (PHA)

Sudan Black B (SBB)

Nile Blue A (NBA)

\begin{abstract}
A B S T R A C T
Polyhydroxyalkanoates (PHA) can be produced and intracellularly accumulated as inclusions by mixed microbial cultures (MMC) for bioplastic production and in enhanced biological phosphorus removal (EBPR) systems. Classical methods for PHA quantification use a digestion step prior to chromatography analysis, rendering them labor intensive and time-consuming. The present work investigates the use of two quantitative image analysis (QIA) procedures specifically developed for PHA inclusions identification and quantification. MMC obtained from an EBPR system were visualized by bright-field and fluorescence microscopy for PHA inclusions detection, upon Sudan Black B (SBB) and Nile Blue A (NBA) staining, respectively. The captured color images were processed by QIA techniques and the image analysis data were further treated using multivariate statistical analysis. Partial least squares (PLS) regression coefficients of 0.90 and 0.86 were obtained between QIA parameters and PHA concentrations using SBB and NBA, respectively. It was found that both staining procedures might be seen as alternative methodologies to classical PHA determination.
\end{abstract}

() 2015 Elsevier B.V. All rights reserved.

\footnotetext{
* Corresponding author. Tel.: +351 253604 407; fax: +351 253604429.

E-mail address: ecferreira@deb.uminho.pt (E.C. Ferreira).
}

\section{Introduction}

Polyhydroxyalkanoates (PHAs) are intracellular storage compounds found in a wide variety of microorganisms under limited nutrient conditions when carbon source is available in surplus [1]. Beyond the important role of PHAs in cell physiology, they are regarded as potential substitutes of traditional petrochemical 
plastics with the additional advantage of being completely biodegradable. PHAs, usually from lipid nature, are used as carbon and energy sources for numerous metabolic synthesis pathways and microbial growth [2]. Nowadays, mixed microbial cultures (MMC) are regarded as alternatives to pure cultures, being able to produce PHAs from renewable resources and without the need for sterile conditions [3-7].

Enhanced biological phosphorus removal (EBPR) systems are commonly used to remove phosphorus from wastewaters. EBPR biomass consists in MMC able to store the substrates intracellularly, in granules or inclusions, and further using them when needed, obtaining a balanced growth with a strong competitive advantage over non storing organisms [8]. In EBPR systems, under anaerobic conditions, MMC take up carbon sources such as volatile fatty acids and store them intracellularly as PHAs and aerobically, their stored PHAs are used as energy source for biomass growth [9].

PHA determination is routinely performed by gas chromatography analysis, with prior digestion, which is a labor intensive and time-consuming methodology, hindering timely results. The search for a faster technique could result in an alternative monitoring procedure to improve EBPR performance.

Quantitative image analysis (QIA) procedures can give valuable information about biological processes. Many applications have been employed specifically in activated sludge (AS) systems for aggregated and filamentous bacteria contents and morphology determination using bright-field and/or phase-contrast [10-13]. The use of fluorescence microscopy has also been reported in the literature in several AS biomass physiological studies (Grampositive/Gram-negative and viable/damaged bacteria ratios) [14].

It is well-known that a number of staining procedures provide quite relevant information for microorganisms' identification. For instance, Gram and Neisser staining are routinely used in MMC for filamentous bacteria characterization and identification [15]. Furthermore, additional procedures include more specialized staining techniques to detect intracellular storage compounds in MMC, such as PHA, poly-P and glycogen, as previously reviewed by Serafim et al. [16] and Mesquita et al. [17]. Nile Red, Nile Blue A (NBA), and Sudan Black B (SBB) are among the most used dyes for selective staining of PHA inclusions [18-22]. However, these staining procedures have been rarely applied in routine analysis, and QIA application has never been considered before as an alternative method to quantify intracellular storage compounds such as PHA.

To overcome the limitations of the standard analytical techniques in intracellular PHA quantification, the present work investigated an advanced monitoring approach based on QIA using SBB and NBA to quantify PHA production from MMC in an EBPR system. Partial least square (PLS) regression models were further applied by integrating image analysis parameters, regarding the biomass structure, alongside classically determined PHA concentration. A comparison between the PHA prediction ability of both staining procedures was also performed.

\section{Materials and methods}

\subsection{Experimental setup and synthetic medium}

A $4 \mathrm{~L}$ sequential batch reactor (SBR) was operated at room temperature (around $20^{\circ} \mathrm{C}$ ), under a 6-h cycle consisting of anaerobic $(120 \mathrm{~min})$, aerobic $(180 \mathrm{~min})$, settling $(55 \mathrm{~min})$, and withdrawing periods $(5 \mathrm{~min})$. The synthetic medium (described below) was added in the first $5 \mathrm{~min}$ of the anaerobic stage. Nitrogen and compressed air were used to ensure anaerobic and aerobic conditions, respectively, using two on/off control valves. The hydraulic retention time (HRT) was $12 \mathrm{~h}$ and the sludge retention time (SRT) was kept at approximately 8 days by wasting mixed liquor at the end of the aerobic stage. Two liters of synthetic medium containing volatile fatty acids (VFA) (solution 1) and phosphate (solution 2) were fed to the system. Solution 1 contained per liter: $2.55 \mathrm{~g} \mathrm{C}_{2} \mathrm{H}_{3} \mathrm{O}_{2} \mathrm{Na} \cdot 3 \mathrm{H}_{2} \mathrm{O}, 0.34 \mathrm{~g} \mathrm{C}_{3} \mathrm{H}_{5} \mathrm{NaO}_{2}$, $0.59 \mathrm{~g} \mathrm{NH}_{4} \mathrm{Cl}, 0.95 \mathrm{~g} \mathrm{MgSO}_{4} \cdot 7 \mathrm{H}_{2} \mathrm{O}, 0.44 \mathrm{~g} \mathrm{CaCl} 2 \cdot 2 \mathrm{H}_{2} \mathrm{O}, 0.0116 \mathrm{~g}$ allyl $-N$ thiourea (to inhibit nitrification), $0.03 \mathrm{~g}$ EDTA, and $3.16 \mathrm{~mL}$ of a trace metals solution. The trace metals solution [23] is listed below $\left(\mathrm{g} \mathrm{L}^{-1}\right)$ : $1.5 \mathrm{FeCl}_{3} \cdot 6 \mathrm{H}_{2} \mathrm{O}, 0.15 \mathrm{H}_{3} \mathrm{BO}_{3}, 0.03 \mathrm{CuSO}_{4} \cdot 5 \mathrm{H}_{2} \mathrm{O}, 0.18 \mathrm{KI}$, $0.12 \mathrm{MnCl}_{2} \cdot 4 \mathrm{H}_{2} \mathrm{O}, \quad 0.06 \quad \mathrm{Na}_{2} \mathrm{MoO}_{4} \cdot 2 \mathrm{H}_{2} \mathrm{O}, \quad 0.12 \quad \mathrm{ZnSO}_{4} \cdot 7 \mathrm{H}_{2} \mathrm{O}$, $0.15 \mathrm{CoCl}_{2} \cdot 6 \mathrm{H}_{2} \mathrm{O}$. Solution 2 contained per liter: $124.1 \mathrm{mg} \mathrm{K} \mathrm{HPO}_{4}$ and $96.8 \mathrm{mg} \mathrm{KH}_{2} \mathrm{PO}_{4}[24]$.

\subsection{Analytical procedures}

The SBR was monitored during 87 days and mixed liquor samples were taken at the end of both anaerobic (AN) and aerobic (AE) stages and centrifuged. The supernatant was discharged and the pellet was lyophilized during at least $48 \mathrm{~h}$. Polyhydroxybutyrate (PHB) and polyhydroxyvalerate (PHV) content was measured by gas chromatography (GC) using the adapted method developed by Smolders et al. [23]. Pre-weighed samples of lyophilized sludge were transferred to glass vials. The polymers were esterified in glass vials with $\mathrm{HCl}$ :1-Propanol and extracted with dichloromethane including benzoic acid as the internal standard. The mixture was vortex-mixed, to promote good contact between the two phases, and was digested at $100^{\circ} \mathrm{C}$ for $3.5 \mathrm{~h}$. After digestion, the content of the vial was transferred with $2 \mathrm{~mL}$ of ultra-pure water to a different vial, covered with a rubber seal, and contact between the two phases was further promoted. The vials were kept in an inverted position for $30 \mathrm{~min}$, after which $1 \mathrm{~mL}$ of the organic phase was collected. Quantification was made in a GC system (Varian 3800 instrument, Varian Inc. USA) equipped with a flame ionization detector. PHB and PHV were separated using a TRWAX capillary column (Teknokroma, Spain), with helium as the carrier gas. The temperatures of the split injection and detector were $220^{\circ} \mathrm{C}$ and $250^{\circ} \mathrm{C}$, respectively. The initial oven temperature was $50^{\circ} \mathrm{C}$ for $2 \mathrm{~min}$, with a $10^{\circ} \mathrm{C} \mathrm{min}^{-1}$ ramp to $225^{\circ} \mathrm{C}$ and then maintained for $10 \mathrm{~min}$. Data was analyzed using the acquisition and integration software Star Chromatography Workstation v. 6.30 (Varian Inc. USA) and calibration curves were obtained for PHB and PHV with an internal standard. In the current work, PHA was determined as the sum of PHB and PHV. Total consumption of acetate and propionate was confirmed at the end of each stage and measured by high performance liquid chromatography (HPLC) using an internal standard. Chromatographic separation was performed using a Varian Metacarb $67 \mathrm{H}$ column $(300 \times 7.8 \mathrm{~mm}$, Varian, USA $)$ under the following conditions: mobile phase $0.005 \mathrm{M} \mathrm{H}_{2} \mathrm{SO}_{4}$, flow rate $0.6 \mathrm{~mL} \mathrm{~min}^{-1}$, and column temperature $60^{\circ} \mathrm{C}$. The system was comprised of a Jasco chromatograph 880-PU intelligent pump (Jasco, Tokyo, Japan) equipped with a Jasco 210-UV detector (Jasco, Tokyo, Japan) and a Jasco AS-2057 Plus intelligent auto sampler (Jasco, Tokyo, Japan). Data was analyzed using the acquisition and integration software Star Chromatography Workstation v. 6.30 (Varian Inc. USA).

\subsection{Staining procedure and bright-field image acquisition}

During the monitoring period, biomass samples were also collected at the end of the $\mathrm{AN}$ and $\mathrm{AE}$ stages. These samples were further homogenized using a tissue grinder, promoting the visualization and image acquisition, and later quantification of PHA intracellular storage polymers by bright-field microscopy. For each sample, a smear of biomass suspension $(100 \mu \mathrm{L})$ was carefully and uniformly deposited on a glass slide and thoroughly air dried. Slides were stained with SBB (0.3\% w/v in 60\% 
Table 1

Image analysis variables from the SBB method used for the PLS analysis of PHA.

\begin{tabular}{ll}
\hline Parameter & Description \\
\hline Predictor $(\mathbf{X})$ & PHA inclusions total area per image \\
& fLn (PHA inclusions total area per image) \\
& PHA inclusions total area per image without border objects \\
& fLn (PHA inclusions total area per image without border objects) \\
& Number of PHA inclusions per image \\
& fExp (number of PHA inclusions per image) \\
& PHA inclusions roundness \\
& PHA inclusions diameter \\
& PHA inclusions perimeter \\
& PHA inclusions length \\
& PHA inclusions width \\
& PHA inclusions form factor \\
& PHA inclusions color intensity per image \\
& fPow (PHA inclusions color intensity per image) \\
Response & Polyhydroxyalkanoates concentration \\
$(\mathbf{Y})$ &
\end{tabular}

fLn represents a logarithmic function, fExp represents an exponential function, and fPow represents a power function.

$\mathrm{v} / \mathrm{v}$ ethanol $)$ for $10 \mathrm{~min}$ and counterstained with safranin $\mathrm{O}(0.5 \%$ $\mathrm{w} / \mathrm{v}$ in deionised water) for $10 \mathrm{~s}$ [15].

Two slides per sample were used and images were acquired in the upper, middle and bottom of each slide, resulting in a total of 150 images $(2 \times 75$ images per slide). Samples were examined by means of an Olympus BX51 (Olympus, Tokyo, Japan) microscope at $1000 \times$ total magnification under oil immersion, and bacteria presenting blue-black color are reported as intracellular PHA accumulating organisms. Images were acquired at $2040 \times 1536$ pixels, and 24-bit RGB format ( 8 bit red, 8 bit green, and 8 bit blue channels) through the commercial software Cell^ ${ }^{\wedge}$ (Olympus, Tokyo, Japan).

\subsection{Image analysis procedure for bright-field images}

The image processing and analysis procedure was based on the identification and quantification of PHA inclusions using a specifically developed program in Matlab 7.8.0 (The Mathworks, Natick, MA). A more detailed description of the image processing methodology is presented below.

The first step of the developed program splits the RGB image into the three composing channels (red, green, and blue). To improve image contrast and increase the definition of the PHA inclusions contours, a logarithmic transformation (natural logarithm followed by normalization) and a contrast enhancement filter (3-by-3 pixels unsharp filter) was performed on the red channel, followed by a 50 pixels kernel size $($ sigma $=20)$ Gaussian filter. This procedure created a blob of uniform color in the image containing all the inclusions and easily segmented by a simple threshold. After this first rough segmentation, an adaptive thresholding algorithm separating the obtained color blob from the background was used. In mixed cultures, the biomass structure can be considerably different from sample to sample, regarding the extensive fraction of flocs, as well as PHA inclusion contents, generally combined as clusters. Thus, to surpass the complexity of image segmentation, color segmentation was performed, removing all the pixels related to the red and green colors, given the fact that SBB stains PHA inclusions in blue-black color. The color segmentation was further based on determining the blue/red and green/blue ratios. It was considered that the pixels related to the red color presented a blue/red ratio below 0.9 , whereas the pixels related to the green color presented a green/blue ratio above 1.1. Afterwards, a 2-by-2 pixels erosion, guided by the brightness, was applied for the individual separation of each PHA inclusion. However, even considering this processing step, some clusters may still be present, mostly due to insufficient color gradient. Therefore, a distance transform based separation was implemented, following a solidity (below 0.8 ) and area (above 100 pixels) criteria for separation. Identified inclusions were post-treated in terms of debris elimination by a 3-by-3 pixels erosion, dilation and filling. In the last processing step, brightness guided erosion (3\% of the brightest pixels) was performed to eliminate inclusions halos.

The segmented PHA inclusions from the collected images were then characterized into the most relevant morphological parameters presented in Table 1. Also presented in Table 1 are a few mathematical transformations of the collected QIA parameters later introduced in the multivariate statistical study.

A schematic representation of the image processing and analysis procedure is shown in Fig. 1 presenting also the original and final binary images.

\subsection{Staining procedure and fluorescence image acquisition}

As it was described in Section 2.3, biomass samples were also collected at the end of the AN and AE stages. The staining procedure for fluorescent image acquisition was quite different from the SBB staining procedure where a smear of the sample was placed on a slide similar to a simple Gram or Neisser staining. In this case a suspension of cells was used, thus, samples were fixed in 4\% formaldehyde, then washed with phosphate buffer saline solution (PBS), and stored in a 50\% PBS - 50\% ethanol solution at $-20^{\circ} \mathrm{C}$ prior to further analysis to preserve the cells as close to its natural state [25].

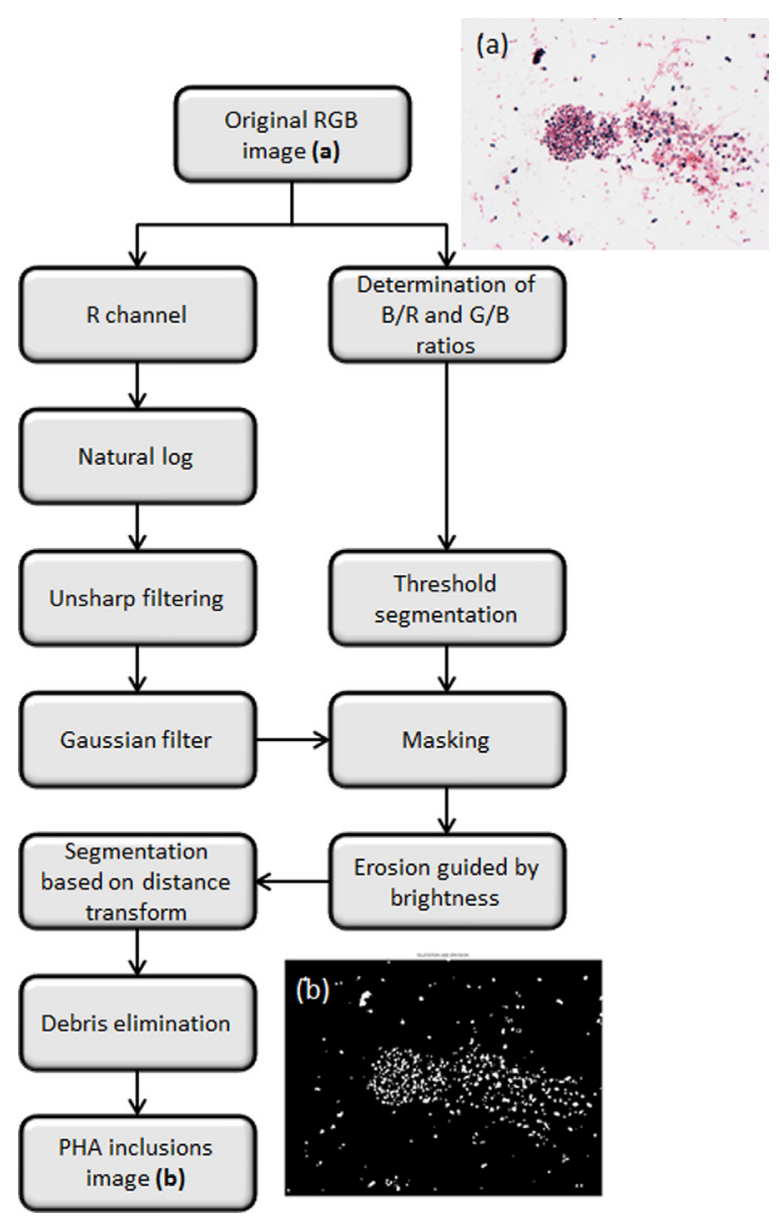

Fig. 1. Schematic representation of the image processing and analysis procedure for SBB staining. 
Table 2

Image analysis variables from NBA method used for the PLS analysis of PHA.

\begin{tabular}{|c|c|}
\hline Parameter & Description \\
\hline Predictor $(\mathbf{X})$ & $\begin{array}{l}\text { Total intensity of the original image } \\
\text { fExp (total intensity of the original image) } \\
\text { Total intensity of the background corrected image } \\
\text { fExp (total intensity of the background corrected image) } \\
\text { PHA inclusions total area per image } \\
\text { fExp (PHA inclusions total area per image) } \\
\text { PHA inclusions equivalent diameter } \\
\text { fExp (PHA inclusions equivalent diameter) } \\
\text { PHA inclusions total intensity calculated from the original image } \\
\text { PHA inclusions average intensity calculated from the original image } \\
\text { fQuad (PHA inclusions average intensity calculated from the original image) } \\
\text { PHA inclusions total intensity calculated from the background corrected image } \\
\text { PHA inclusions average intensity calculated from the background corrected image } \\
\text { fQuad (PHA inclusions average intensity calculated from the background corrected image) }\end{array}$ \\
\hline
\end{tabular}

Response (Y)

Polyhydroxyalkanoates concentration

fExp represents an exponential function, and fQuad represents a quadratic function.

For fluorescence image acquisition, and for each sample, $1 \mathrm{~mL}$ of fixed cell suspension was incubated at $55^{\circ} \mathrm{C}$ for 10 min with 1 drop of NBA according to the methodology described in Ostle and Holt [26], and further centrifuged at $4500 \mathrm{rpm}$ for $5 \mathrm{~min}$ as described in Mesquita et al. [21]. Pellet cells were washed with $0.9 \% \mathrm{NaCl}$ and centrifuged using the same conditions. The excess of stain solution was removed using $8 \%$ acetic acid for $1 \mathrm{~min}$ and centrifuged again. Pellet cells were then re-suspended in $0.9 \% \mathrm{NaCl}$. In order to guarantee good quality images for the implementation of QIA, a tissue grinder was used to homogenize the aggregates formed during the staining procedure.

Three slides per sample were used, and for each slide a volume of $10 \mu \mathrm{L}$ of the stained sludge samples was covered with a $20 \mathrm{~mm} \times 20 \mathrm{~mm}$ cover slip, for visualization and image acquisition. Images were acquired in the upper, middle, and bottom of the slide resulting in a total of 150 images ( $3 \times 50$ images per slide). The slides were examined by means of an Olympus BX51 (Olympus, Tokyo, Japan) fluorescence microscope at $400 \times$ total magnification, with constant exposure times for intensity measurements [21]. A long pass filter was used with an excitation wavelength of $530-550 \mathrm{~nm}$ and emission cut off at $591 \mathrm{~nm}$, with the stained PHA inclusions presenting an emission spectra around $625-675 \mathrm{~nm}$, for the employed excitation wavelength. Images were acquired at $1360 \times 1024$ pixels, and 24-bit RGB format ( 8 bit red, 8 bit green, and 8 bit blue channels) through the commercial software Cell ${ }^{\wedge} \mathrm{B}$ (Olympus, Tokyo, Japan).

\subsection{Image analysis procedure for fluorescence images}

The image processing and analysis methodology regarding PHA inclusions was based on the identification and quantification of PHA inclusions regions using a specifically developed program in Matlab 7.8.0 (The Mathworks, Natick, MA). A more detailed description of the image processing methodology is presented below.

The first step of the developed program splits the RGB image into the three composing channels (red, green, and blue). Only the red channel was subsequently used for the remaining treatment, given the fact that NBA stained PHA emitted at a red spectral wavelength. A background correction step, by means of tophat filtering (100 pixels size) was next applied, followed by Wiener filter smoothing. The identification of the PHA regions was performed in a two fold manner: for well and poorly defined inclusion regions. For the identification of well-defined inclusion regions a top hat filter ( 30 pixels size) was applied followed by edge determination and filling, whereas for the poorly defined inclusion regions a threshold based segmentation was applied. Both identified inclusion regions were then combined in a single image further post-treated for debris elimination by morphological erosion and reconstruction. The final binary image was then used for the determination of the total PHA region area and subsequently used as a mask image in both the original red channel image and the background corrected red channel image.

The recognized PHA inclusions from the resulting images were then characterized into the most relevant morphological parameters described in Table 2. Also presented in Table 2 are a few mathematical transformations of the collected IA parameters later introduced in the multivariate statistical study.

A schematic representation of the image processing and analysis procedure is shown in Fig. 2 presenting also the original and final binary images.

\subsection{Multivariate statistical analysis}

In partial least squares (PLS) regression, the decomposition of $\mathbf{X}$ and $\mathbf{Y}$ is carried out iteratively. By exchanging information between the two blocks in each step, the latent variables (LVs) of the $\mathbf{X}$-space are rotated so that the predictive power of the $\mathbf{X}$-space with regard to the $\mathbf{Y}$-space is enhanced [27]. Matrix (X) was always preprocessed using the standard normal variate method to remove undesirable variations. Matrix $\mathbf{Y}$ values were used both for model development and to test its performance. In PLS it is critical to determine the optimal number of LVs and cross-validation (CV) is a reliable way to test the predictive significance of each PLS regression. For that purpose, part of the training dataset is kept out of the model, predicted and finally compared with the actual values (using $\mathrm{CV}$ ). A more detailed explanation of the PLS algorithm could be found elsewhere [28-31]. However, it was found that the number of LVs depicted by this methodology did not allow an acceptable training model. Thus, for selecting the optimal number of LVs, a random $2 / 3$ training dataset to $1 / 3$ validation dataset was repeated 20,000 times to obtain a total number of 20,000 randomly selected different datasets. The cumulative fraction of the Y explained by the components (R2Y(sum)), the root mean squared error of prediction (RMSEP) and the correlation coefficient $\left(R^{2}\right)$ between measured and modelgenerated $Y$ values was then determined for the training set. For each of the 20,000 different datasets, these parameters were determined for a number of LVs ranging from one to half the number of $X$ variables in the model. Furthermore, the correlation coefficient $\left(R^{2}\right)$ between measured and model-generated Y values was also determined for the overall (comprehending both training and validation) dataset, and used as the selection criteria to choose the best model. 


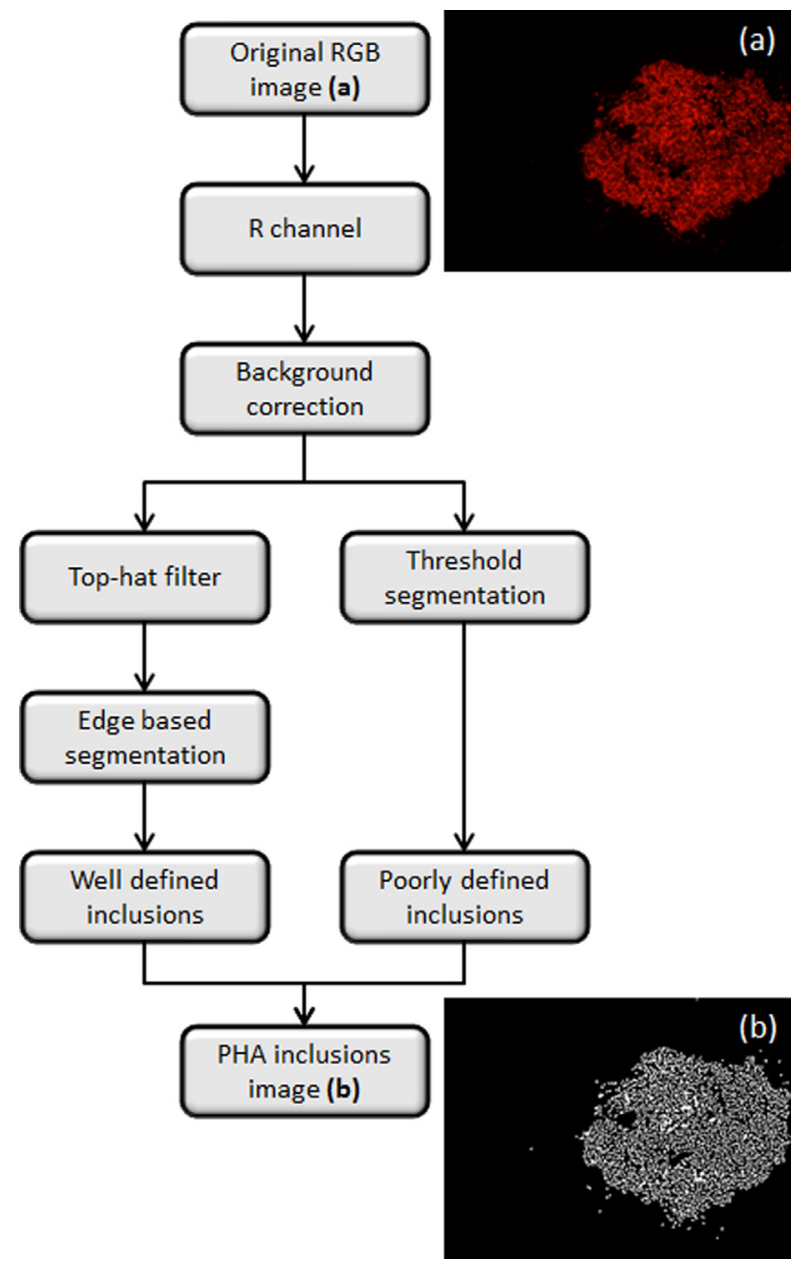

Fig. 2. Schematic representation of the image processing and analysis procedure for NBA staining.

The software Matlab ${ }^{\mathrm{TM}}$ 7.8.0 (The Mathworks, Natick, MA) was used for predicting PHA by PLS with a total of 134 samples from SBB and NBA data. A total of 64 samples (32 AN and $32 \mathrm{AE}$ ) belonged to the $\mathrm{SBB}$ dataset whereas the remaining 70 samples (35 AN and $35 \mathrm{AE}$ ) belonged to the NBA dataset. Four studies were performed using different datasets regarding the obtained QIA results for the SBB, and another four for the NBA datasets. For both, a first PLS model was conducted using the global QIA results, consisting of the ensemble of AN and $A E$ stage datasets (further designated as global). The second PLS model was comprised of only the AN data, whereas the third PLS model was comprised of only the AE stage.
Furthermore, in the fourth study (further designated as combined or $\mathrm{AN}+\mathrm{AE}$ ), the independent PLS results of the $A N$ and AE data were combined together after independent PLS analyses. Data were randomly divided into a training set $(67 \%$ of the observations) to train the model, and a validation set (33\% of the observations) to validate the model.

Similar PLS analyses were conducted with a prior crosscorrelation (CC) analysis of the image analysis collected data to reduce the dataset (matrix $\mathbf{X}$ ), leading to the exclusion of one variable per pair presenting a correlation factor above 0.9 . In the case of the SBB dataset, PHA inclusions total area per image, PHA inclusions diameter, PHA inclusions perimeter, PHA inclusions width and fLn (PHA inclusions total area per image) parameters were excluded. In the case of the NBA dataset, total intensity of the original image, PHA inclusions total intensity calculated from the original image, PHA inclusions total intensity calculated from the background corrected image, PHA inclusions average intensity calculated from the background corrected image and fQuad (PHA inclusions average intensity calculated from the original image) parameters were excluded from the original matrix $\mathbf{X}$. The number of $\mathrm{X}$ variables prior and after $\mathrm{CC}$ analysis was 14 and 9 variables for both the SBB and NBA datasets.

\section{Results and discussion}

In this work, the ability of using QIA data from SBB and NBA staining to predict intracellular storage PHA through PLS analysis was pursued. For this purpose, two datasets from the lab-scale EBPR system experiments were used. The first dataset used QIA information provided by bright-field microscopy and SBB staining (Table 1), whereas the second dataset used QIA information provided by fluorescent microscopy and NBA staining (Table 2). A standard analytical methodology was also employed to determine intracellular PHA concentrations as previously reported in Section 2.2. For both methodologies, samples were obtained at the end of the anaerobic and aerobic stages.

The first results of PLS models, using the global aerobic and anaerobic QIA data, were performed with the ensemble AN (anaerobic) and AE (aerobic) datasets. The PLS analysis results (latent variables (LVs), cumulative fraction of the Y explained by the components (R2Y(sum)), root mean squared error of prediction (RMSEP), regression coefficients $\left(R^{2}\right)$ and linear regression equations) for PHA prediction (with and without CC analysis), for the training, validation and overall (training+validation) datasets are depicted in Table 3. Each regression coefficient and equation corresponds to the linear correlation found by the least squares method. The predicted results of the intracellular PHA concentrations, for both SBB and NBA staining without CC analysis, were somewhat distant from 1 (correlation coefficient $R^{2}$ of 0.78 and 0.73 , respectively). However, concerning the prediction

Table 3

PLS prediction results for PHA using the global anaerobic (AN) and aerobic (AE) image analysis data with and without cross-correlation (CC).

\begin{tabular}{|c|c|c|c|c|}
\hline & \multicolumn{2}{|l|}{ SBB } & \multicolumn{2}{|l|}{ NBA } \\
\hline & $\begin{array}{l}\text { Without CC } \\
\text { (14 variables) }\end{array}$ & $\begin{array}{l}\text { With CC } \\
(9 \text { variables })\end{array}$ & $\begin{array}{l}\text { Without CC } \\
\text { (14 variables) }\end{array}$ & $\begin{array}{l}\text { With CC } \\
\text { (9 variables) }\end{array}$ \\
\hline LVs & 7 & 4 & 7 & 4 \\
\hline R2Y (cum) & 0.83 & 0.82 & 0.80 & 0.70 \\
\hline RMSEP & 6.41 & 6.05 & 5.52 & 7.92 \\
\hline$R^{2}$ training set & 0.80 & 0.79 & 0.77 & 0.61 \\
\hline Linear regression (training set) & $y=0.93 x$ & $y=0.93 x$ & $y=0.93 x$ & $y=0.90 x$ \\
\hline$R^{2}$ validation set & 0.77 & 0.63 & 0.71 & 0.66 \\
\hline Linear regression (validation set) & $y=1.08 x$ & $y=1.06 x$ & $y=1.11 x$ & $y=1.31 x$ \\
\hline$R^{2}$ overall (training + validation) set & 0.78 & 0.73 & 0.73 & 0.55 \\
\hline Linear regression (training + validation set) & $y=0.97 x$ & $y=0.96 x$ & $y=0.97 x$ & $y=0.98 x$ \\
\hline
\end{tabular}


Table 4

PLS prediction results for PHA using independent anaerobic (AN) and aerobic (AE) image analysis data with and without cross-correlation (CC).

\begin{tabular}{|c|c|c|c|c|c|c|c|c|}
\hline & \multicolumn{4}{|l|}{ SBB } & \multicolumn{4}{|l|}{ NBA } \\
\hline & \multicolumn{2}{|c|}{$\begin{array}{l}\text { Without CC } \\
\text { (14 variables) }\end{array}$} & \multicolumn{2}{|c|}{$\begin{array}{l}\text { With CC } \\
\text { (9 variables) }\end{array}$} & \multicolumn{2}{|c|}{$\begin{array}{l}\text { Without CC } \\
\text { (14 variables) }\end{array}$} & \multicolumn{2}{|c|}{$\begin{array}{l}\text { With CC } \\
\text { (9 variables) }\end{array}$} \\
\hline & AN & $\mathrm{AE}$ & AN & $\mathrm{AE}$ & AN & $\mathrm{AE}$ & AN & $\mathrm{AE}$ \\
\hline LVs & 7 & 7 & 4 & 4 & 7 & 7 & 4 & 4 \\
\hline R2Y (cum) & 0.92 & 0.93 & 0.86 & 0.92 & 0.95 & 0.88 & 0.70 & 0.90 \\
\hline RMSEP & 5.8 & 6.1 & 6.1 & 5.2 & 6.0 & 6.4 & 9.0 & 8.7 \\
\hline $\mathrm{R}^{2}$ training set & 0.91 & 0.92 & 0.84 & 0.91 & 0.95 & 0.87 & 0.60 & 0.90 \\
\hline Linear regression (training set) & $y=0.98 x$ & $y=0.97 x$ & $y=0.96 x$ & $y=0.97 x$ & $y=0.98 x$ & $y=0.95 x$ & $y=0.90 x$ & $y=0.96 x$ \\
\hline$R^{2}$ validation set & 0.87 & 0.91 & 0.68 & 0.91 & 0.68 & 0.79 & 0.79 & 0.66 \\
\hline Linear regression (validation set) & $y=0.97 x$ & $y=1.11 x$ & $y=0.95 x$ & $y=1.12 x$ & $y=1.01 x$ & $y=1.15 x$ & $y=1.14 x$ & $y=1.00 x$ \\
\hline$R^{2}$ overall (training + validation) set & 0.89 & 0.91 & 0.78 & 0.90 & 0.88 & 0.83 & 0.63 & 0.85 \\
\hline Linear regression (training + validation set) & $y=0.97 x$ & $y=1.00 x$ & $y=0.96 x$ & $y=1.00 x$ & $y=0.99 x$ & $y=1.00 x$ & $y=0.96 x$ & $y=0.97 x$ \\
\hline$(\mathrm{AN}+\mathrm{AE}) R^{2}$ (training + validation set) & 0.90 & & 0.84 & & 0.86 & & 0.75 & \\
\hline Linear regression $(\mathrm{AN}+\mathrm{AE})$ & $y=0.99 x$ & & $y=0.98 x$ & & $y=1.00 x$ & & $y=0.96 x$ & \\
\hline
\end{tabular}

ability of NBA staining, it is important to notice a marked improvement with respect to previously obtained results [21], from a correlation coefficient $R^{2}$ of 0.40 to $R^{2}$ of 0.73 , indicating that the QIA methodology was further enhanced.

With respect to the use of the CC analysis for dataset reduction, for both SBB and NBA staining, quite lower prediction abilities were obtained ( $R^{2}$ of 0.73 and $R^{2}$ of 0.55 , respectively). Given these results, data reduction was found to be unadvisable.

To improve the prediction ability for intracellular PHA concentrations, for both SBB and NBA, a different set of studies was performed, in which each of the two sampling periods (AN and AE) was modeled independently by PLS analysis. The results of the two independent analyses were then merged to obtain the combined $(\mathrm{AN}+\mathrm{AE})$ PHA concentration predictions. The PLS analysis results (latent variables (LVs), cumulative fraction of the Y explained by the components (R2Y(sum)), root mean squared error of prediction (RMSEP), regression coefficients $\left(R^{2}\right)$ and linear regression equations) for PHA prediction of this study are depicted in Table 4. The training, validation, and overall (training + validation) for the AN and $\mathrm{AE}$ stages, and combined $(\mathrm{AN}+\mathrm{AE})$ results are presented. Again, the regression coefficients and regression equations correspond to the linear correlations found by the least squares method.

Comparing the PHA prediction ability from the AN and AE stages calculated independently, without CC analysis, the PLS results demonstrated good homogeneity between the SBB and NBA results. In fact, the overall (training + validation) regression coefficient $\left(R^{2}\right)$ varied solely from 0.83 to 0.91 and the linear slope was very close to 1.00 . Thus, and despite the slightly better results for the SBB staining, both methodologies were found to be appropriate for PHA quantification. Furthermore, this was further emphasized with the results obtained by combining the two independent analyses ( $\mathrm{AN}+\mathrm{AE}$ ) with regression coefficients of 0.90 and 0.86 and slopes of 0.99 and 1.00 , for SBB and NBA respectively. The comparison between the independent PLS results $(\mathrm{AN}+\mathrm{AE})\left(R^{2}\right.$ of 0.90$)$ and the overall dataset results (Table 3$)$ of the first study ( $R^{2}$ of 0.78 ), showed a marked improvement for the SBB dataset. Fig. 3 shows the correlation between the measured and predicted values for intracellular PHA concentrations, for $(A N+A E)$ independent PLS results for NBA and SBB, resulting in a similar correlation for both staining procedures. For the NBB dataset an

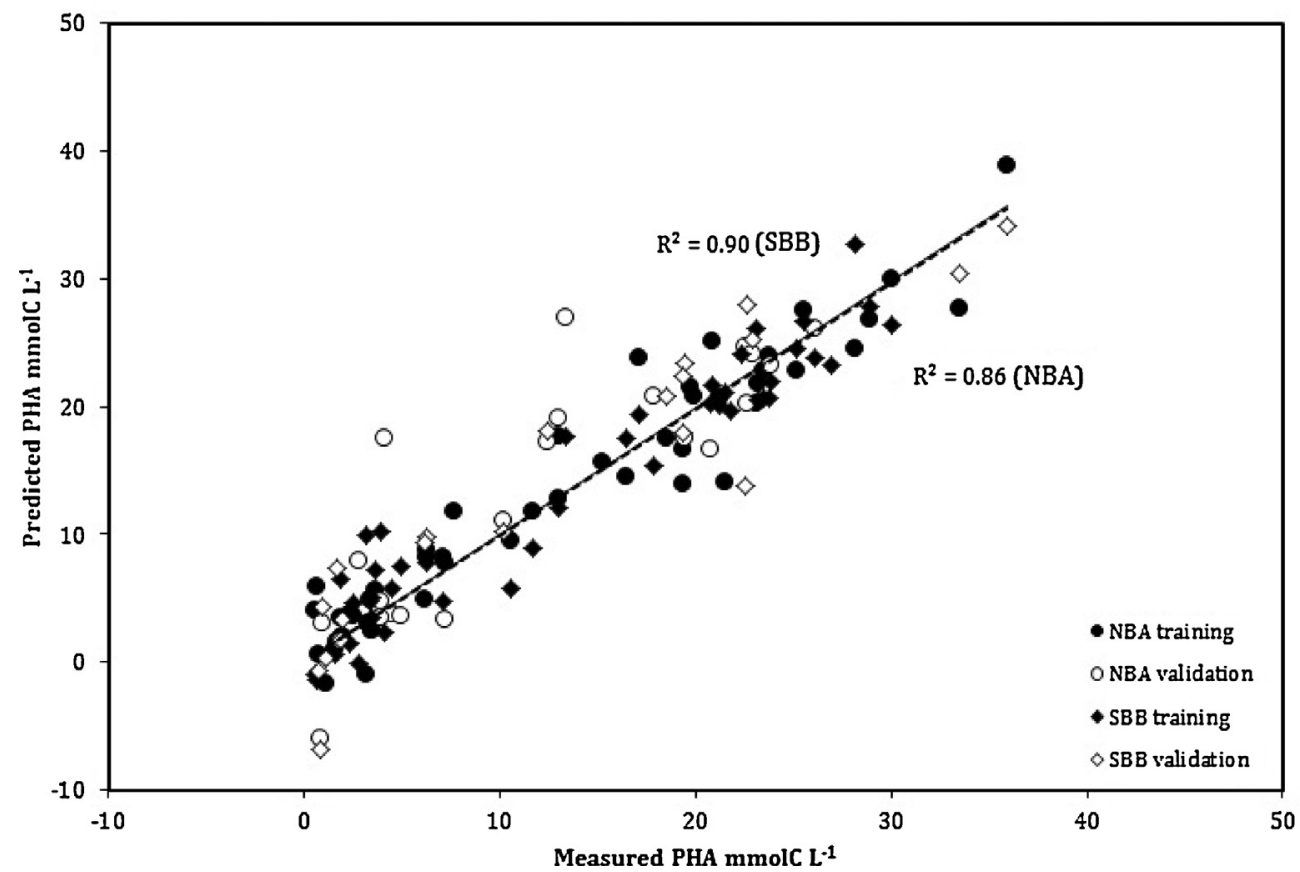

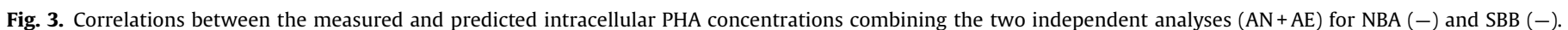


even greater improvement on the prediction ability was obtained for the independent PLS results $(\mathrm{AN}+\mathrm{AE})\left(R^{2}\right.$ of 0.86$)$ when compared to the overall dataset results from Table $3\left(R^{2}\right.$ of 0.73$)$. These results highlight the importance of performing independent analyses on the data collected from the aerobic and anaerobic stages.

It has been already stated that EBPR systems work using a strategy that combines fully AN feast and AE famine cycle using MMC. Within this strategy, PHA enrichment is performed in the AN stage, when an external carbon source is available. In this stage, and according to previous research by Oehmen et al. [32,33], a prompt storage of PHA is generally achieved. It is also known that the PHA accumulated in the AN stage can be used as a carbon/ energy reserve in substrate starvation in the following AE stage $[32,33]$. In this case, a sharp increase in PHA consumption is frequently observed within this specific stage of the biological process. Thus, the use of a larger range of PHA data, combining both $\mathrm{AE}$ and AN results, would be expected to increase the PLS prediction ability. However, this was not the case in the current work, perhaps since the range of PHA concentrations in the AN and AE stages are similar, given the fact that the PHA levels are low at the beginning of the AN stage and increase to their maximum value at the end of the stage, while in the AE stage PHA decreases from this high level achieved at the end of the AN stage until very low levels. Further research investigation is warranted to determine the underlying cause for the difference in PLS prediction ability using the global and independent data sets.

It should be stressed also that, the PLS model results regarding NBA staining were markedly improved when compared to prior results [21], from a correlation coefficient $R^{2}$ of 0.65 to $R^{2}$ of 0.86 , indicating that the QIA methodology has been significantly enhanced. Again, it was found that a prior CC analysis considerably hindered the PHA prediction ability in all cases. In fact, significantly lower prediction abilities $\left(R^{2}\right.$ of 0.84 and $R^{2}$ of 0.75 for SBB and NBA datasets, respectively) were obtained with CC analysis, further indicating that data reduction was found to be unadvisable for this study.

In conclusion, both SBB and NBA staining procedures can be seen as valuable methodologies to predict intracellular PHA storage inclusions, provided that independent aerobic and anaerobic PLS analyses are performed.

\section{Conclusions}

In the current work, bright-field and fluorescent staining, on one hand, and QIA methodologies on the other, were studied and validated to predict intracellular PHA intracellular storage inclusions. It was found that SBB and NBA staining were able to predict well the PHA concentrations using independent anaerobic and aerobic data treatment, which was found to be fundamental with the studied EBPR samples. Furthermore, this study emphasized the usefulness of SBB and NBA staining, coupled to QIA methodologies, in the future research of PHA production from mixed microbial cultures involved in wastewater treatment.

\section{Acknowledgements}

The authors thank the Fundação para a Ciência e a Tecnologia (FCT) Strategic Project PEst-OE/EQB/LA0023/2013 and the Project "BioEnv - Biotechnology and Bioengineering for a sustainable world”, REF. NORTE-07-0124-FEDER-000048, co-funded by the Programa Operacional Regional do Norte (ON.2 - O Novo Norte), QREN, FEDER. The authors also acknowledge the financial support to Daniela P. Mesquita through the postdoctoral grant (SFRH/BPD/ $82558 / 2011$ ) and the project PTDC/EBB-EBI/103147/2008 provided by FCT.

\section{References}

[1] S.R.K. Pandiana, V. Deepak, K. Kalishwaralal, J. Muniyandi, N. Rameshkumar, S, Gurunathan, Synthesis of PHB nanoparticles from optimized medium utilizing dairy industrial waste using Brevibacterium casei SRKP2: a green chemistry approach, Colloids Surf. B 74 (2009) 266-273.

[2] R.C. Fuller, Microbial inclusions with special reference to PHA inclusions and intracellular boundary envelopes, Int. J. Biol. Macromol. 25 (1999) 21-29.

[3] M.A.M. Reis, L.S. Serafim, P.C. Lemos, A.M. Ramos, F.R. Aguiar, M.C.M. Van Loosdrecht, Production of polyhydroxyalkanoates by mixed microbial cultures, Bioprocess Biosyst. Eng. 25 (2003) 377-385.

[4] G. Carvalho, A. Oehmen, M.G.E. Albuquerque, M.A.M. Reis, The relationship between mixed microbial culture composition and PHA production performance from fermented molasses, N. Biotechnol. 31 (2014) 257-263.

[5] A.F. Duque, C.S.S. Oliveira, I.T.D. Carmo, A.R. Gouveia, F. Pardelha, A.M. Ramos, M.A.M. Reis, Response of a three-stage process for PHA production by mixed microbial cultures to feedstock shift: impact on polymer composition, N. Biotechnol. 31 (2014) 276-288.

[6] M.G.E. Albuquerque, C.A.V. Torres, M.A.M. Reis, Polyhydroxyalkanoate (PHA) production by a mixed microbial culture using sugar molasses: effect of the influent substrate concentration on culture selection, Water Res. 44 (2010) 3419-3433.

[7] M.G.E. Albuquerque, V. Martino, E. Pollet, L. Averous, M.A.M. Reis, Mixed culture polyhydroxyalkanoate (PHA) production from volatile fatty acid (VFA)-rich streams: effect of substrate composition and feeding regime on PHA productivity, composition and properties, J. Biotechnol. 151 (2011) 66-76.

[8] M.C.M. Van Loosdrecht, M.A. Pot, J.J. Heijnen, Importance of bacterial storage polymers in bioprocesses, Water Sci. Technol. 35 (1997) 41-47.

[9] A. Oehmen, P.C. Lemos, G. Carvalho, Z.G. Yuan, J. Keller, L.L. Blackall, M.A.M Reis, Advances in enhanced biological phosphorus removal: from micro to macro scale, Water Res. 41 (2007) 2271-2300.

[10] M. da Motta, M.N. Pons, N. Roche, Automated monitoring of activated sludge in a pilot plant using image analysis, Water Sci. Technol. 43 (2001) 91-96.

[11] M. da Motta, M.N. Pons, N. Roche, H. Vivier, Characterisation of activated sludge by automated image analysis, Biochem. Eng. J. 9 (2001) 165-173.

[12] R. Jenné, E.N. Banadda, I.Y. Smets, A. Bamelis, L. Verdickt, J.F. Van Impe Activated sludge image analysis system: monitoring settleability and effluent clarity, Water Sci. Technol. 52 (2005) 193-199.

[13] A.L. Amaral, E.C. Ferreira, Activated sludge monitoring of a wastewater treatment plant using image analysis and partial least squares regression, Anal. Chim. Acta 544 (2005) 246-253.

[14] D.P. Mesquita, A.L. Amaral, E.C. Ferreira, Identifying different types of bulking in an activated sludge system through quantitative image analysis, Chemosphere 85 (2011) 643-652.

[15] D. Jenkins, M.G. Richard, G.T. Daigger, Manual on the Causes and Control of Activated Sludge Bulking and Foaming, WRC, Pretoria and USEPA, Cincinnati, 2003.

[16] L.S. Serafim, P.C. Lemos, C. Levantesi, V. Tandoi, H. Santos, M.A.M. Reis, Methods for detection and visualization of intracellular polymers stored by polyphosphate-accumulating microorganisms, J. Microbiol. Methods 51 (1) (2002) 1-18.

[17] D.P. Mesquita, A.L. Amaral, E.C. Ferreira, Activated sludge characterization through microscopy: a review on quantitative image analysis and chemometric techniques, Anal. Chim. Acta 802 (2013) 14-28.

[18] K.L. Burdon, Fatty material in bacteria and fungi revealed by staining dried, fixed slide preparations, J. Bacteriol. 52 (1946) 665-678.

[19] G. Redzwan, S.N. Gan, I.K.P. Tan, Isolation of polyhydroxyalkanoate-producing bacteria from an integrated-farming pond and palm-oil mill effluent ponds, World J. Microbiol. Biotechnol. 13 (1997) 707-709.

[20] Z. Alias, I.K.P. Tan, Isolation of palm oil-utilising, polyhydroxyalkanoate (PHA)producing bacteria by an enrichment technique, Bioresour. Technol. 96 (2005) 1229-1234.

[21] D.P. Mesquita, C. Leal, J.R. Cunha, A. Oehmen, A.L. Amaral, M.A.M. Reis, E.C. Ferreira, Prediction of intracellular storage polymers using quantitative image analysis in enhanced biological phosphorus removal systems, Anal. Chim. Acta 770 (2013) 36-44.

[22] D.P. Mesquita, G. Selvaggio, J.R. Cunha, C. Leal, A.L. Amaral, E.C. Ferreira, Image analysis for automatic characterization of polyhydroxyalcanoates granules, Lect. Notes Comput. Sci. 7950 (2013) 790-797.

[23] G.J.F. Smolders, J. van de Meij, M.C.M. van Loosdrecht, J.J. Heijnen, Model of the anaerobic metabolism of the biological phosphorus removal process: stoichiometry and pH influence, Biotechnol. Bioeng. 43 (1994) 461-470.

[24] M. Carvalheira, A. Oehmen, G. Carvalho, M.A.M. Reis, The effect of substrate competition on the metabolism of polyphosphate accumulating organisms (PAOs), Water Res. 64 (2014) 149-159.

[25] G. Wallner, R. Amann, W. Beisker, Optimizing fluorescent in situ hybridization with rRNA-targeted oligonucleotide probes for flow cytometric identification of microorganisms, Cytometry 14 (1993) 136-143.

[26] A.G. Ostle, J.G. Holt, Nile Blue A as a fluorescent stain for poly-betahydroxybutyrate, Appl. Environ. Microbiol. 44 (1982) 238-241.

[27] C. Rosen, A Chemometric Approach to Process Monitoring and Control with Applications to Wastewater Treatment Operation. PhD Thesis, Lund Institute of Technology, Sweden, 2001. 
[28] K.P. Singh, N. Basant, A. Malik, G. Jain, Modeling the performance of up-flow anaerobic sludge blanket reactor based wastewater treatment plant using linear and nonlinear approaches-a case study, Anal. Chim. Acta 658 (2010) 1-11.

[29] P. Teppola, S.P. Mujunen, P. Minkkinen, Partial least squares modeling of an activated sludge plant: a case study, Chemom. Intell. Lab. Syst. 38 (1997) 197-208.

[30] S.H. Woo, C.O. Jeon, Y.S. Yun, H. Choi, C.S. Lee, D.S. Lee, On-line estimation of key process variables based on kernel partial least squares in an industrial cokes wastewater treatment plant, J. Hazard. Mater. 161 (2009) 538-544.
[31] J.W. Einax, H.W. Zwanziger, S. Geiss, Chemometrics in Environmental Analysis, VCH, Weinheim, Germany, 1997.

[32] A. Oehmen, Z. Yuan, L.L. Blackall, J. Keller, Comparison of acetate and propionate uptake by polyphosphate accumulating organisms and glycogen accumulating organisms, Biotechnol. Bioeng. 91 (2005) 162-168.

[33] A. Oehmen, A.M. Saunders, M.T. Vives, Z. Yuan, J. Keller, Competition between polyphosphate- and glycogen-accumulating organisms in enhanced biological phosphorus removal systems with acetate and propionate as carbon sources, J. Biotechnol. 123 (2006) 22-32. 